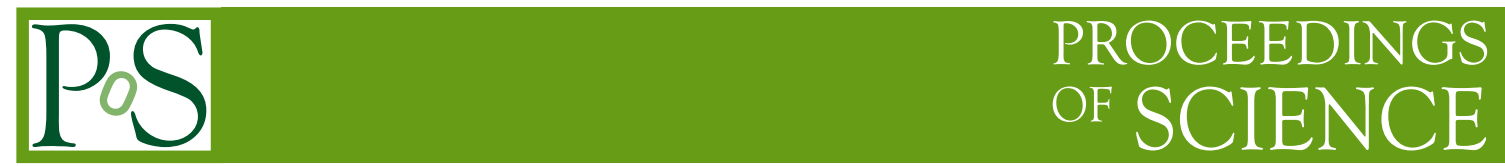

\title{
What have we learned? Outstanding questions...
}

\section{Philip Kaaret*}

Department of Physics and Astronomy, University of Iowa, Iowa City, IA 52242, USA

Université Paris 7 Denis Diderot and Service d'Astrophysique, UMR AIM, CEA Saclay, F-91191

Gif sur Yvette, France

E-mail: philip-kaaret@uiowa.edu

I highlight a few general themes about blazars and their variability discussed in this workshop and then raise some outstanding open questions that will, hopefully, be answered in a future workshop.

Workshop on Blazar Variability across the Electromagnetic Spectrum April 22-25 2008

Palaiseau, France

${ }^{*}$ Speaker. 


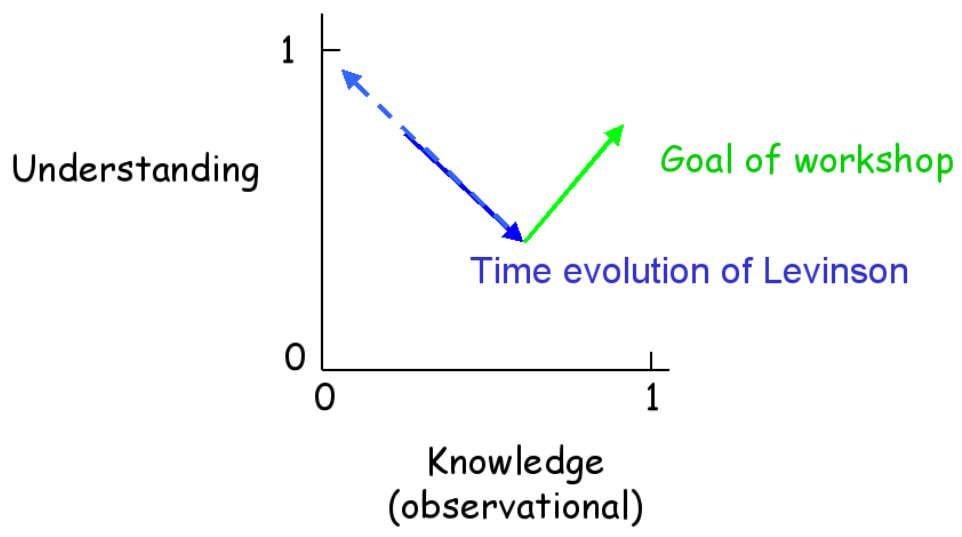

Figure 1: Time evolution of Levinson based on his comment about blazars that "the more I know about these objects, the less I understand them". The solid line decreasing with time shows the time evolution of Levinson. The dashed line shows an extrapolation backwards in time which may have been the motivation of the organizers in choosing the rapporteur. The solid line increasing with time shows the goal of the workshop: to change the sign of the time evolution of Levinson.

\section{Introduction}

Those who know me well, or who have at least have looked me up on the NASA ADS service, realize that I have made few contributions to the literature on blazars. ${ }^{1}$ An honest assessment of my knowledge of blazars would place it close to zero. Thus, it was with some amusement that I considered and ultimately accepted the request of the workshop organizers to give the summary talk. However, during the opening session of the meeting, Amir Levinson made a comment which may illuminate the thinking of the scientific organizing committee. Levinson said, referring to blazars, that "the more I know about these objects, the less I understand them". Fig. 1 shows the time evolution of Levinson. If one extrapolates the evolution of Levinson backwards in time, then one can estimate the level of understanding of a person with near zero knowledge of blazars and, thus, a possible motivation for the committee's choice of rapporteur. Interestingly, the same plot also gives a graphical representation of the goal of the workshop: to change the sign of the time evolution of Levinson.

There were a great many interesting results presented at this workshop. One of the advantages of a printed review talk is that it is not necessary to cover them all since the readers may simply have a look at the table of contents and then read the papers themselves. The many excellent results presented give encouragement that our understanding of blazars really does increase with our observational knowledge. In the following, I attempt to highlight a few general themes and raise some outstanding open questions.

\section{What are we studying?}

Study of blazars focuses mainly on the broad spectrum of radiation produced by the jet. But

\footnotetext{
${ }^{1}$ A good review talk starts with humor in order to re-awaken the audience. Such humor doesn't often translate well to the printed page, so it is with great trepidation that I reproduce the opening comments of my talk here.
} 


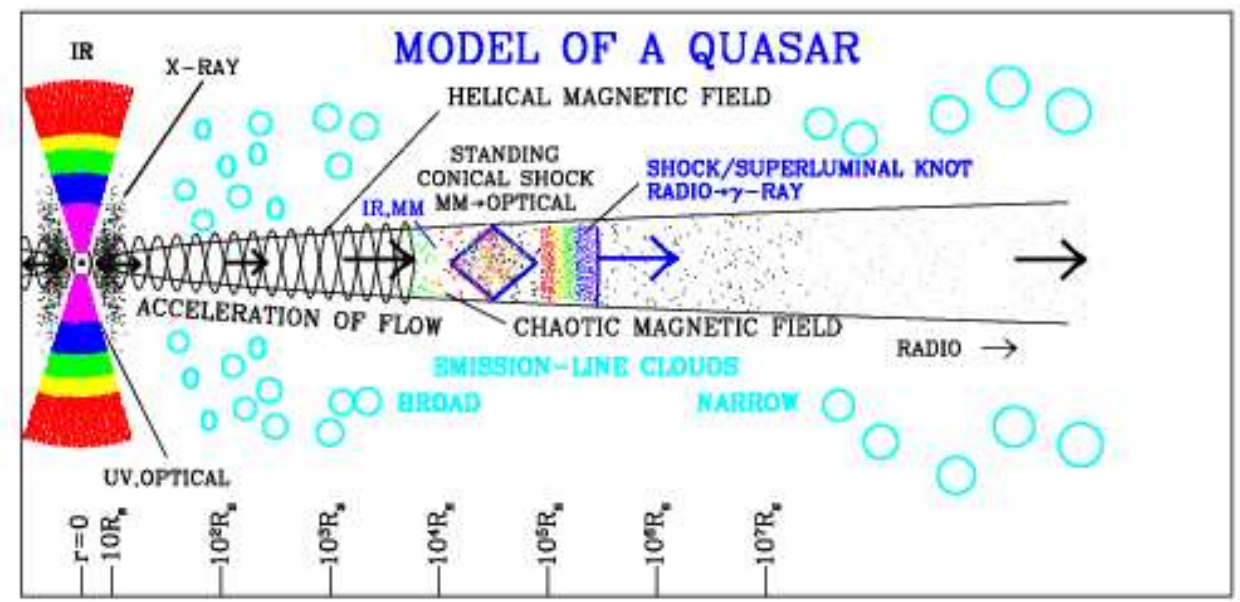

Figure 2: The inner regions of the accretion disk produce a 'blob' of energy and/or matter that is then accelerated in the jet and produces radiation. Figure is from Marscher.

where does variability seen in the jet originate? It is useful to consider our physical picture of blazars at the most basic level as a starting point in our attempts to understand the origins of the observed variability. A much used diagram from Marscher is reproduced in Fig. 2. Physically, we believe that the inner regions of the accretion disk produce a 'blob' of energy and/or matter that is then accelerated (the black hole spin may help push) in the jet and produces radiation. Thus, variability may arise in the jet or be already present in the input from the accretion disk to the jet.

In one of the most provocative ${ }^{2}$ talks of the meeting, McHardy suggested that much, if not all, of the the variability observed from blazars originates in the accretion disk. To examine this idea, it is useful to think of the jet as an electrical circuit. Input to the circuit arises from the accretion disk. The circuit then acts on the input and produces an output. If the circuit is linear, then the jet variability would be determined exactly by the disk input. In this case, the variability would be the same at all wavelengths. This is not observed. Indeed, the variability of blazars is markedly different in the different parts of the electromagnetic spectrum. Thus, the suggestion that the action of the jet is linear and all of the observed variability arises from the disk can be rejected. The jet itself must be a non-trivial source of variability.

However, as discussed by McHardy, accretion disks are complex systems and are well known sources of variability on a wide range of time scales. Specifically, the variability of accretion disks is known to have a red noise distribution and a log-normal distribution of amplitudes. Some of the variability observed from the jet is produced in the disk. Realistic blazar variability modeling must start with injection of 'blobs' (or a continuous stream of matter and energy) with a red noise power spectrum and a log normal amplitude distribution to simulate the disk input. This has already been done in some simulations, but should be adopted generally. The study of blazars is primarily the study of processes occurring in the jet. However, it is key that the models begin with the known noise properties of accretion disks as input.

\footnotetext{
${ }^{2}$ Indeed, this was the only talk where bodily harm to the speaker was discussed during lunch as a logical conclusion to the presentation.
} 

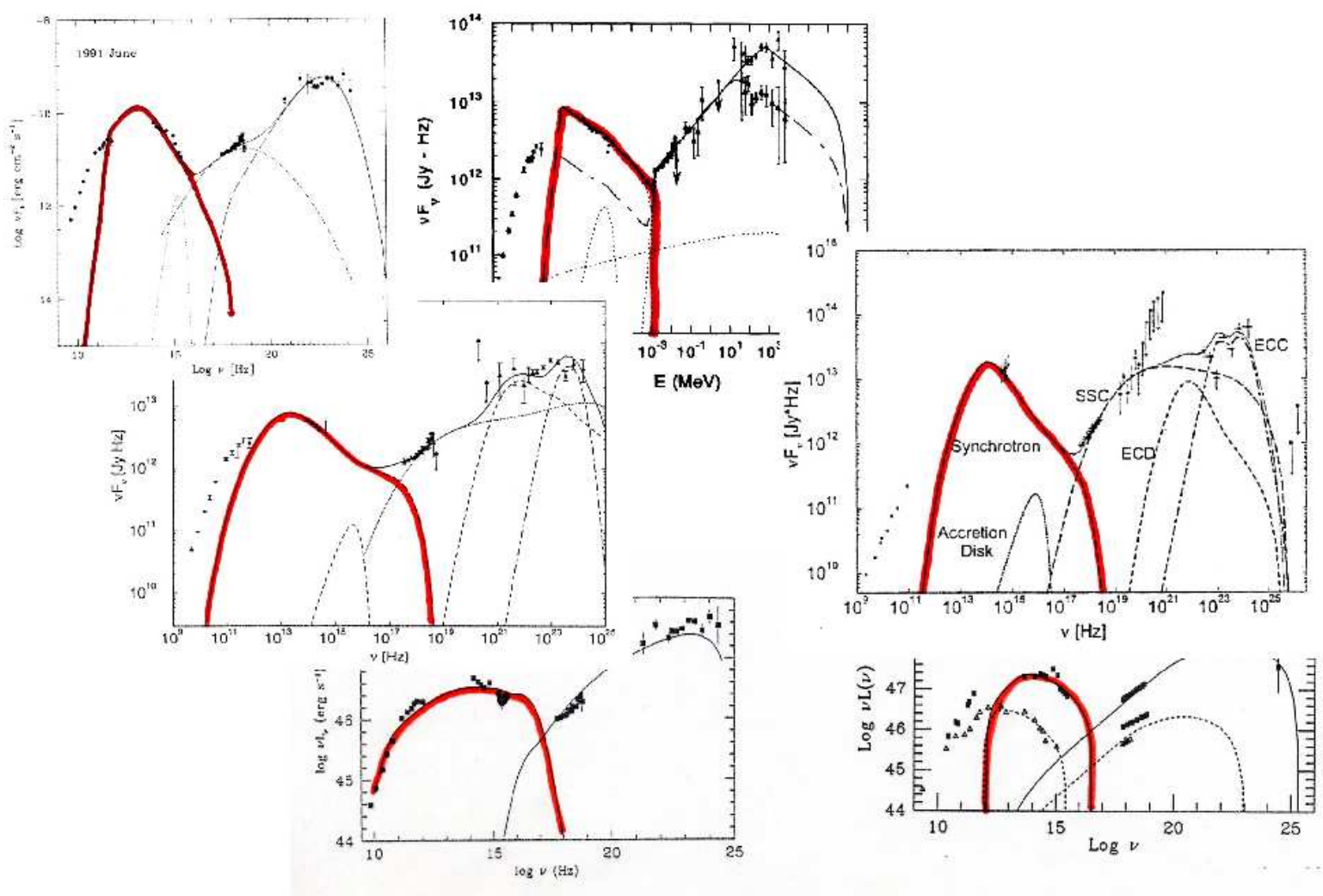

Figure 3: Six different spectral energy distribution models fit to the same set of multiwavelength data obtained for 3C 279 in June 1991 from Valtaoja.

\section{The need for time dependent models}

My favorite view graph of the meeting was shown by Valtaoja, see Fig. 3, and shows six very different spectral energy distribution models fit to the same set of multiwavelength data obtained for 3C 279 in June 1991. The figure succinctly demonstrates the fact that individual 'snapshots' of the spectral energy distribution of blazars are insufficiently constraining to uniquely identify the underlying physical mechanisms acting in blazars. Since time variability is the next logical step, the figure also provides strong motivation for the basic idea of this meeting: that study of time variability is essential to understand blazars.

A further caution regarding the use of individual spectral energy distributions (SEDs) was raised by Henri. A common feature of most (essentially all) blazar jet models is that radiation at different wavelengths arises from different locations in jet. Thus, the instantaneous SED measured in our reference frame likely arises from spatially separated regions. Fitting of an individual SED in terms of a single population of energetic particles may be misleading because the energy distribution may evolve as particles move through the jet. Indeed, if the particle energy distribution does vary temporally, and thus spatially, it may be impossible to reproduce individual instantaneous 
SEDs with a single particle population.

Time dependent modeling is clearly essential for understanding the physics of blazars. This necessarily increases the complexity of the modeling, particularly when the need to use an appropriate input noise spectrum from the accretion disk is taken into account, but a number of groups have already risen to the challenge. The need to consider time dependence also raises observational issues, particularly in the design and execution of multiwavelength campaigns.

\section{Designing a multiwavelength campaign}

Reliable inferences about correlations between the emission observed at difference wavelengths can be made only if the monitoring duration is many times longer than the correlation delay and the typical flare duration ${ }^{3}$. Some correlation delays reported at this workshop include: an Xray/optical lag of $\sim 20$ days reported for 3C279 by Marscher, an X-ray/radio lag of $114 \pm 21$ days reported for 3C279 by Marscher, a TeV/X-ray lag of less than 30 minutes reported for PKS 2155304 by Benbow, a hard X-ray/soft X-ray lag of about 300 minutes reported for 1ES 1218+304 by Sato and Kataoka. Although these correlation delays have been found only for individual sources and, thus, may not be applicable for all blazars, the trends suggest that long duration campaigns are needed when comparing optical or radio emission with X-ray or gamma-ray emission, while short duration campaigns should suffice when comparing X-ray with gamma-ray emission.

It is important to note that the correlation delay time scales between a given pair of wavebands (say X-ray versus optical) may depend on the spectral energy distribution of the object. For example, wavebands which are correlated on short time scales for high-energy peaked blazars may be correlated on much longer time scales for low-energy peaked blazars.

Given that a typical multiwavelength campaign runs over a few months at most, an interest conclusion from the correlation time scales mentioned above is that optical and radio are better done as dedicated monitoring programs than as part of a limited duration multiwavelength campaign. The correlation time scales are simply too long to be adequately characterized by a multiwavelength campaign of one to two month duration. Fortunately, many groups appear to have risen to the task of conducting dedicated monitoring programs in the optical. Some of the groups described by Tosti and in the various posters include: Perugia, Colgate, Turin, Rome, Turku, Rem at La Silla, Georgia State, Calar Alto, WEBT, and KANATA/Trispec. Radio monitoring was less well covered at this meeting with representation by the groups from Tuorla-Metsähovi (Valtaoja, Hovatta, Lindfors, Nieppola) and Boston University (Jorstad, Marscher). Coverage is also needed in the southern hemisphere.

In the high energy range, the recent launch of the GLAST gamma-ray observatory should revolutionize GeV gamma-ray coverage of blazars (Madejski). The observing strategy of GLAST, to scan the whole sky every three hours, is ideal for monitoring a large number of GeV bright blazars. GLAST will also be an important source of triggers for target of opportunity observations with X-ray satellites and $\mathrm{TeV}$ observatories. Due to the short correlation times between X-rays and gamma-rays, relatively short duration, triggered multiwavelength campaigns should suffice to study the correlations between these bands. The study of bright flares is adequate as long as the

\footnotetext{
${ }^{3}$ This point is particularly tricky if the noise has a red noise spectrum with power at very long time scales.
} 
statistical nature of the underlying process is understood as described by Degrange. The Whipple $\mathrm{TeV}$ telescope has been dedicated to monitoring a small number of blazars known to exhibit bright $\mathrm{TeV}$ flares. The DWARF project would construct a similar, small telescope for $\mathrm{TeV}$ monitoring of a handful of bright blazars.

A resource for coordination of blazar monitoring and multiwavelength campaigns is the Blazar multiwavelength Wiki hosted by the Adler Planetarium at castor.adlerplanetarium.org/MWL. The wiki provides a means to inform others in the community about your observations and is not intended as a data repository. Sharing of data and publication rights would be negotiated between individuals or institutions after contact is made based on information obtained from the wiki.

\section{Questions: big and little}

Some of the big questions regarding blazars are:

- How are jets formed and collimated?

- Are jets electron/hadron or electron/positron?

- Are jets purely accretion powered or can jets extract energy from the rotation of a black hole?

- What is the role of magnetic fields?

- Can particles be accelerated to TeV energies without a jet?

These questions are sufficiently general that they apply equally well to non-blazar AGN and stellar-mass black hole binaries. Progress on any of these questions would be a significant step forward in the broad study of accreting black holes.

While the big questions are broad and important, it may be more useful to focus near term research on a set of more specific 'little' questions. Progress on these questions should be possible in the short term and answers to these questions would be a significant step forward in our understanding of blazar jets. Answering some or all of these little questions is likely prerequisite to addressing the big questions. Some of the 'little' questions raised in this workshop include:

- What is the magnitude of the magnetic field in jets? What is the configuration of the magnetic field in jets?

- What is the average luminosity of a given blazar?

- What fraction of the accretion power goes into jets? (Perlman)

- Can blazar light curves be decomposed using a universal shape? Is the shape the same for different energies? What can we learn from the shape?

- Do TeV flares really have very high Doppler factors?

- Do (some) particles thermalize at the shock front (Kirk)

- Should we develop new timing tools?

- How can we resolve the question of the composition of the jets?

In the following, I discuss a few of these questions in more detail.

\section{What is the magnetic field in jets?}

Numbers for the magnetic field in blazars jets mentioned during the various talks in this workshop range from $100 \mu \mathrm{G}$ to $100 \mathrm{G}$, a range of only six orders of magnitude. However, before 


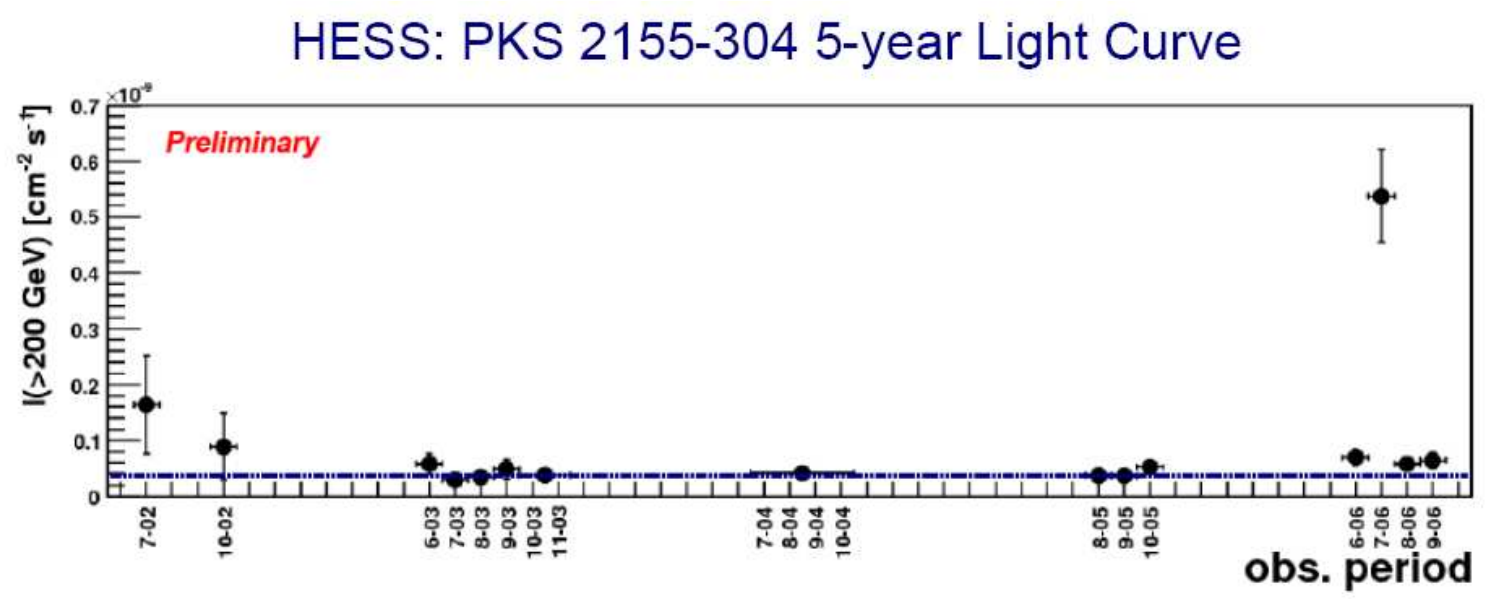

Figure 4: TeV light curve of PKS 2155-304 as measured with HESS from Benbow.

attempting to assign a unique number to the magnetic field, it is useful to consider the structure of the jet and the magnetic field. In a structured jet, e.g. one with a fast moving core and a slower sheath, the magnetic field is likely to be strongly radially dependent. Thus, any measurement of the magnetic field must also address the question of where within the jet the radiation used to make the measurement is produced.

Several techniques to measure magnetic fields were described in the various talks. One interesting method is use of the X-ray flare shape as described by Kataoka. They observed an X-ray flare from 1ES 1218+304 in which the hard X-ray emission (5-10 keV) lagged behind the softer $\mathrm{X}$-ray emission. This sign of lag is opposite that usually observed from blazars, and may occur only when the electrons producing the hard X-ray emission are close to the maximum of the distribution. By fitting the hard lag as a function of energy, the product of the magnetic field multiplied by the ratio of the energy in the ordered versus turbulent magnetic fields can be determined. It would be of interest to search for similar flares from other blazars, particularly those in which the electrons of maximum energy produce hard X-rays. Also, the simple model used does not accurately reproduce the flare rise times at low energies and additional modeling may be needed. Other techniques presented for the measurement of the magnetic field magnitude and geometry include measurement of the turnover frequencies in sub-mm/infrared (Mannheim), optical circular polarization, and radio polarization (Gabuzda). Modeling of spectral energy distributions is, perhaps, the most common technique used, but until time-dependent models and extensive multiwavelength monitoring is available, progress on the most direct techniques in individual wavebands may lead to quicker progress.

\section{What is the average luminosity of a given blazar?}

This, seemingly trivial, question is raised by the light curve of PKS 2155-304 presented by Benbow, see Fig. 4. The TeV emission of PKS 2155-304 is generally at a very low level, but very large and very rare flares are sometimes (well, once) observed. Since broad-band spectral energy distributions are used for tasks as basic as classifying blazars, how one should construct those SEDs is of critical importance. For example, should we construct the blazar sequence using the peak or average luminosities? The relative magnitudes of the synchrotron versus Compton peaks for PKS 


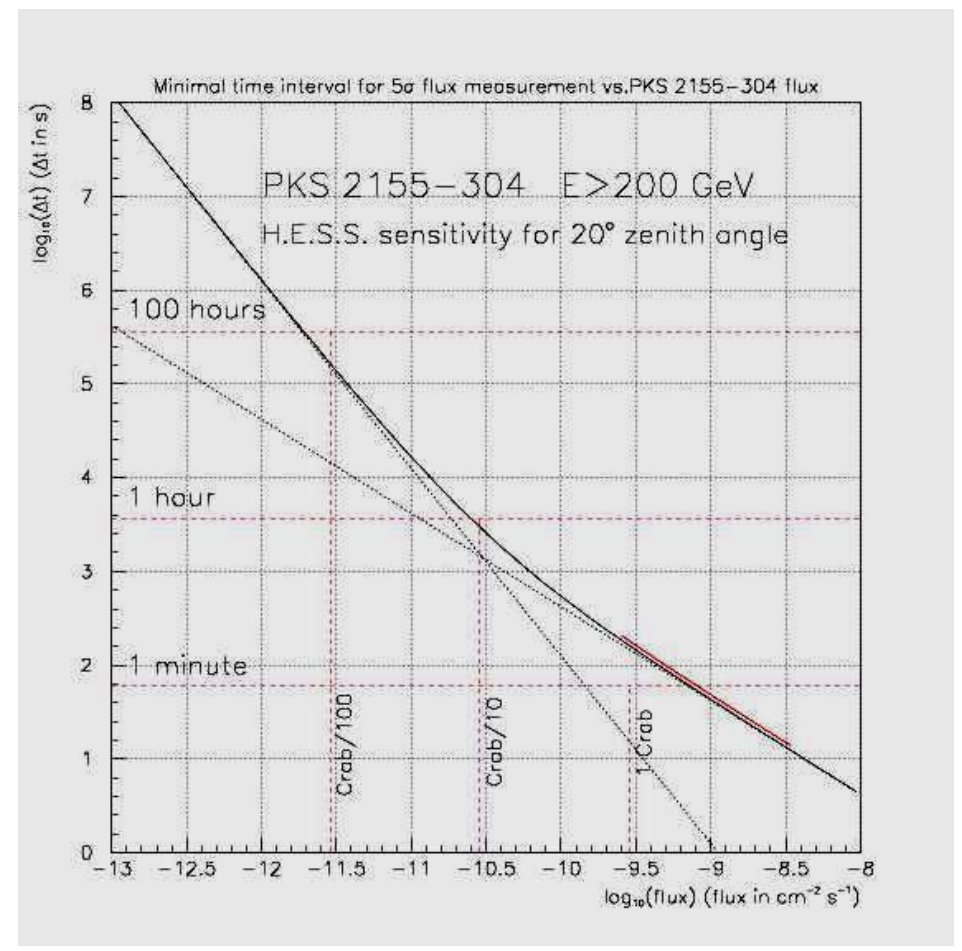

Figure 5: Minimum time for a flux measurement at the 5- $\sigma$ level versus source flux for TeV observations of PKS 2155-304 using HESS from Degrange.

2155-304 depend strongly on whether the quiescent or peak TeV flux is used. Thus, whether or not a given blazar is classified as Compton dominant or not may depend on when it is observed. Another question regards the duty factors of blazars in various energy bands. This is well understood at optical and longer wavelengths, but is poorly known at shorter wavelengths. Long term monitoring is essential to resolve this question. GLAST will make an very important contribution at the high energy end and more sensitive X-ray all-sky monitors such as MAXI (Kataoka) are needed.

\section{Should we develop new timing tools?}

The roots of most mathematical techniques used to quantify variability lie in the theory of continuous functions and their initial applications were typically to regularly sampled time series of measurements. Thus, when doing timing analysis on a individual observation, one typically first bins the data into uniform bins. This is far from the optimum situation for blazar studies at high energies where one has highly variable fluxes and photon counting experiments. As illustrated by Fig. 5, from Degrange, the minimum time needed to obtain a flux measurement varies with the source flux. Thus, uniform binning applied to an observation of a flare where the flux varies with time must balance losing significance in bins with low flux against losing information on rapid variability at times with high flux. It is possible to construct timing tools that use variable-sized time bins or the individual photon arrival times (such as the Rayleigh test for periodicity). As discussed by Degrange, Wagner, and Madejski, development of such tools may allow us to better exploit current and future observations of blazars in the high energy wavebands. 